\title{
High-throughput phenotyping of cell-to-cell interactions in gel microdroplet pico-cultures
}

Juliette Ohan ${ }^{1,3}$, Benjamin Pelle', Pulak Nath², J-H Huang' , Blake Hovde', Momchilo Vuyisich', Armand EK Dichosa' \& Shawn R Starkenburg *,1,4

\section{ABSTRACT}

Microbiomes exert significant influence on our planet's ecology. Elucidating the identities of individual microbes within these communities and how they interact is a vital research imperative. Using traditional plating and culturing methods, it is impractical to assess even a small fraction of the interactions that exist within microbial communities. To address this technology gap, we integrated gel microdroplet technology with microfluidics to generate millions of microdroplet cultures (MDs) that sequester individual cells for phenotyping MDs, facilitating rapid analysis and viable recovery using flow cytometry. Herein, we describe a validated high-throughput phenotyping pipeline that elucidates cell-to-cell interactions for millions of combinations of microorganisms. Through iterative co-culturing of an algae and a pool of environmentally sourced microbes, we successfully isolated bacteria that improved algal growth.

\section{METHOD SUMMARY}

A high-throughput method to identify cell-to-cell interactions within complex microbial communities was designed and validated. This platform integrates gel microdroplet and microfluidic technologies with flow cytometry to enable the identification and recovery of viable cells with specific phenotypes.

\section{KEYWORDS:}

algae $\cdot$ emulsion $\cdot$ encapsulation $\cdot$ flow cytometry $\cdot$ high-throughput $\cdot$ microdroplet - microfluidics $\cdot$ picoculture

'Bioscience Division, Los Alamos National Laboratory, Los Alamos, NM 87545, USA; ${ }^{2}$ Physics Division, Los Alamos National Laboratory, Los Alamos, NM 87545, USA; ${ }^{3}$ Department of Microbiology, Oregon State University, Corvallis, OR 97330 , USA; ${ }^{4}$ New Mexico Consortium, Los Alamos, NM 87544, USA; *Author for correspondence: shawns@ lanl.gov

BioTechniques 66: 218-224 (May 2019) 10.2144/ btn-2018-0124
Cell-to-cell interactions are ubiquitous in nature. Whether planktonic or in biofilms, the human gut or environmental wastewater, microbes thrive in diverse communities. Cell-to-cell interactions within these communities can be extremely complex, and may involve chemical signalling, lateral gene transfer, interspecies competition, predation, syntrophy and/or allelopathy [1]. The classical approach to understand microbial community activity is to isolate individual strains in pure culture; however, $>99 \%$ of microbes are difficult to grow axenically [2]. One microbial community can be composed of tens of thousands of microbes and, therefore, comprise several millions of individual interactions that underlie overall community function. Even if these microbes were readily cultivated, standard monoculture-based approaches fail to characterize all the microbial interactions and interspecies dependencies that define community structure and overall function.

Microfluidic droplet-based emulsion cultures [3] can be used to generate large numbers of individual cell suspensions, which can be analyzed within a reasonable time frame and materials cost. It is possible to create millions of picoliter-sized aqueous droplets or 'pico-cultures' surrounded by a layer of biocompatible oil using droplet generators and microfluidic particle manipulations $[4,5]$. These pico- to nano-liter volume droplets provide individual, isolated environments to grow cells. Current droplet-based microfluidic technology capabilities include deterministic encapsulation [4,6], pico-injections [7], and droplet sorting [8]. Some labs have developed systems that integrate droplet generation, analysis of cell contents via staining, and selective droplet retrieval in one chip $[9,10]$. However, these commercial platforms are highly specialized for specific assays and do not simultaneously enable highthroughput cultivation, screening and recovery of microorganisms exhibiting multiple phenotypes. It is possible to carry out the initial steps of this process using rapid prototyping techniques for microfluidic platforms [11,12]. However, selection and nondestructive isolation of the droplets containing pico-cultures of interest requires the integration of sophisticated optics and electronics.

Fluorescence-activated cell sorting (FACS) instruments are typically accessible at most academic and industrial institutions, and can be exploited to directly enrich cells exhibiting a particular phenotype. However, FACS cannot be used to directly sort microdroplets (MDs) enveloped in oil because the instruments are incompatible with nonaqueous suspensions. To sort oil-covered MDs using FACS, it is necessary to carry out an additional emulsification step, which is often difficult and time-consuming, to generate water-in-oil-in-water double emulsions [13]. Even then, there are inherent limitations associated with flow sorting typical microfluidic droplets under high fluidic shear conditions while maintaining cell viability. One approach to circumvent these shortcomings is to encapsulate cells in gel microdroplets (GMDs) [14,15], as an alternative to surrounding the cells with oil.

GMDs are composed of agarose that can encapsulate one or several microorganisms. The gel matrix of the GMD is porous, thereby providing flow of nutrients, metabolic wastes, and gases to and from the encapsulated cells. Cells or microorganisms can grow into clonal microcolonies that remain conveniently packaged within the GMD enclosure. Previous biological applications of GMDs have included isolation of antibodies [16], pico-liter volume biochemical reactions [17] and cell isolation for single-cell genomics [18-20]. GMDs 
$\checkmark$ have also been implemented in microfluidic platforms where millions of chemically isolated cultures can be formed by directly suspending them in biocompatible oil $[21,22]$. However, suspending cell-laden GMDs directly in an oil phase limits long-term culturing, which is required to develop cellto-cell interactions from complex microbiomes. To enable long-term culturing within GMDs in a microfluidic platform, we investigated encapsulating the GMDs inside an aqueous droplet, suspended in biocompatible oil. Using this technique, it is possible to provide additional nutrients in the aqueous droplet while maintaining chemically distinct culturing environments. Furthermore, it is possible to break the emulsion to recover intact GMDs and maintain cell viability after removing the oil.

Herein, we report a new technique to enable high-throughput investigations of microbial interactions; high-throughput screening of cell-to-cell interactions (HiSCl) (Figure 1). HiSCl exploits commercially available tools and technologies to facilitate maturation of interspecies interactions. Using $\mathrm{HiSCl}$, we demonstrate that individual bacteria species can be identified and recovered from a complex microbial community that enhance the growth of Chlorella sorokiniana, a candidate algal biofuel production strain [23].

\section{MATERIALS \& METHODS}

Encapsulation of algae \& bacteria cells in GMDs

Bacterial suspensions were co-captured with an algae, C. sorokiniana (DOE 1412). Bacteria were aseptically collected and pooled from freshwater and sediment samples in $50 \mathrm{ml}$ conical tubes from various environmental sources (ponds, streams, riverbanks, etc.) in New Mexico. Samples were filtered through a $5 \mu \mathrm{m}$ filter to remove debris and particulates, gently vortexed, and stored at $30^{\circ} \mathrm{C}$ prior to encapsulation in GMDs. Algal and bacterial cells were captured in GMDs (OneCell Systems, Inc.) using our previously described protocol [20] with a few modifications (described in detail in the Supplementary data).

\section{Enrichment of algal GMDs \& algal/ bacterial GMDs with FACS}

Detailed methods to select algal GMDs (aGMDs) and algal/bacterial GMDs (abGMDs) containing the desired cellular content and phenotype are described in the Supplementary data and Supplementary Figure 1. Briefly, a three-gate strategy was developed whereby the GMDs were initially flowanalyzed using forward scatter (FSC) versus side scatter (SSC) to identify the GMDs containing algal and bacterial cells. The first enrichment gate was used to exclusively analyze and identify GMDs that contained algal chlorophyll $a$ and $b$ fluorescence using filters $650 / 50$ versus SSC. The, GMDs within the chlorophyll subgate were analyzed using $650 / 50$ versus SSC to target aGMDs and abGMDs with the desired cell number. GMDs sorted under this gating strategy resulted in sorting of abGMDs with one algal cell (>80\%) per GMD, while the sorted abGMDs possessed one to five putative bacterial cells per GMD.

\section{Packaging of GMDs into MDs \\ for culturing}

GMDs were encapsulated into picolitersized MDs using a microfluidic droplet generator (Figure 2), as described in the supplemental material. After the GMDs were packaged into MDs, they were transferred via sterile syringes to a sealed, optically transparent cultivation chamber (Supplementary Figure 2). The cultivation chamber enables visualization on a standard microscope stage and facilitates even light distribution. Silicone tubes leading to the inlet chambers were cut leaving behind $\sim 5 \mathrm{~cm}$ of tubing, and sealed with plastic clamps. The sealed cultivation chamber was placed on an orbital shaker (110 rpm) and incubated at $22^{\circ} \mathrm{C}$ for at least 7 days to facilitate growth of algae and/or bacteria inside the GMDs. The cultivation time was optimized for Chlorella to limit growth to 3-4 doublings inside the GMDs.

\section{MD destabilization \& recovery of GMDs}

After the culturing period, the MD emulsion was collapsed to recover GMDs. In brief, the GMD/MD oil emulsion was removed from the cultivation chamber and destabilized as described in the supplemental material. This process collapsed the emulsion, resulting in a visible oil phase and an aqueous GMD phase. The resultant aqueous layer was transferred to a sterile $5 \mathrm{ml}$ polypropylene tube for FACS processing.
Selection of GMDs with desired

phenotypes using FACS

FACS was applied to enrich the aGMDs and abGMDs with microcolonies (post-cultivation), using conditions identical to those described above, but with a modified gating strategy. A sub-gate in SSC versus FSC to an initial chlorophyll autofluorescence gate was used to select for abGMDs containing algae and to measure changes in growth/ expansion of the algal microcolonies (Supplementary Figure 3). Using this approach, 500 target abGMDs were sorted into a microcentrifuge tube and visually confirmed via microscopy to contain an algal microcolony (Figure 3B micrograph). Microcolony growth in a subpopulation of the abGMDs demonstrates that some fraction of the algae originally encapsulated in the abGMDs (est. $n \leq 5 / G M D$ ) remained viable throughout the cultivation pipeline and, thus, could be recovered for downstream applications.

\section{MD stability \& analyte exchange}

MDs were prepared as described above using: (1) sterile high salt (HS) growth medium alone; and (2) sterile HS medium containing Alexa Fluor 532 fluorescent dye. The two preparations were combined and incubated in a microfluidic cultivation chamber for 60 days at $22^{\circ} \mathrm{C}$. After incubation, the MDs were analyzed under a brightfield microscope to assess droplet stability and size homogeneity. Concurrently, fluorescence microscopy was used to determine if the Alexa Fluor dye had transferred between MDs.

\section{Bacterial isolation \& identification}

Pooled and filtered samples were extracted for microbial DNA using the Quick-DNA Fungal/Bacterial Kit (Zymo research) and identified by sequencing the PCR-amplified V3/V4 hypervariable regions of the 16S rRNA gene using Illumina MiSeq [24,25]. Strains were isolated by plating on HS media [26] amended with $1.5 \%$ agar $(15 \mathrm{~g} / \mathrm{l})$. The metagenome of the bacterial population post-iterative screening was sequenced using the Illumina Miseq platform. To characterize the population of organisms present after each previous round of $\mathrm{HiSCl}$ screening, QIIME [27] was utilized to cluster each V3/V4 16S rRNA gene region paired end data and cluster combined reads into 
operational taxonomic units (OTUs). QIIME parameters were set to the following: read quality score cut-off of q29, OTU sequence similarity threshold of 0.96 , and a minimum number of OTU sequences per cluster of two. Taxonomic assignments of each OTU was assigned within QIIME using the Greengenes 16 S reference database [28]. Readbased metagenomic organism identification was performed using GOTTCHA [29] using default settings. Sequences were deposited in GenBank (Table 1).

\section{Bacterial co-culture \&}

\section{phenotypic validation}

A bacterial strain isolated from the iterative screening process, Pseudomonas spp., was co-cultured with $C$. sorokiniana 1412 to confirm the veracity of the growth-promoting phenotype. Five replicates containing $100 \mathrm{ml}$ of HS growth medium in 250-ml Erlenmeyer flasks were inoculated with $C$. sorokiniana 1412 with and without the bacterial isolate. All cultures were incubated in a $16 \mathrm{~h}$ light and $8 \mathrm{~h}$ dark cycle, under constant shaking at $45 \mathrm{rpm}$, for a period of 19 days.

\section{RESULTS \& DISCUSSION}

To validate the HiSCI workflow, an environmentally sourced pool of bacteria was packaged with algae inside GMDs, which were then packaged in individual MDs and cultured for 7 days. Microcolony growth of the algae was observed in the GMDs via microscopy (e.g., Figure 3B micrograph). With HiSCl, cells are randomly captured in GMDs as a function of Poisson distribution, thus the average number of cells in each GMD is dependent on the density of the input cell suspension. We estimated that an input algal cell suspension with an $\mathrm{OD}_{750}$ $\sim 3.4\left(\sim 4.0 \times 10^{6}\right.$ cells) would, on average, result in the capture of one algal cell per GMD [20]. Even under optimized conditions many GMDs remain empty following the cell encapsulation step. Thus, the enrichment step (Figure 1, step 3) is crucial to improve the efficiency of the workflow. Using our FACS approach for GMD enrichment, 330,900 potential abGMDs were collected prior to MD encapsulation (Figure 3A and Supplementary figure 1).

To validate MD stability, two separate MD batches, prepared with and without a fluorophore, were combined and monitored for exchange/dilution of the fluorophore

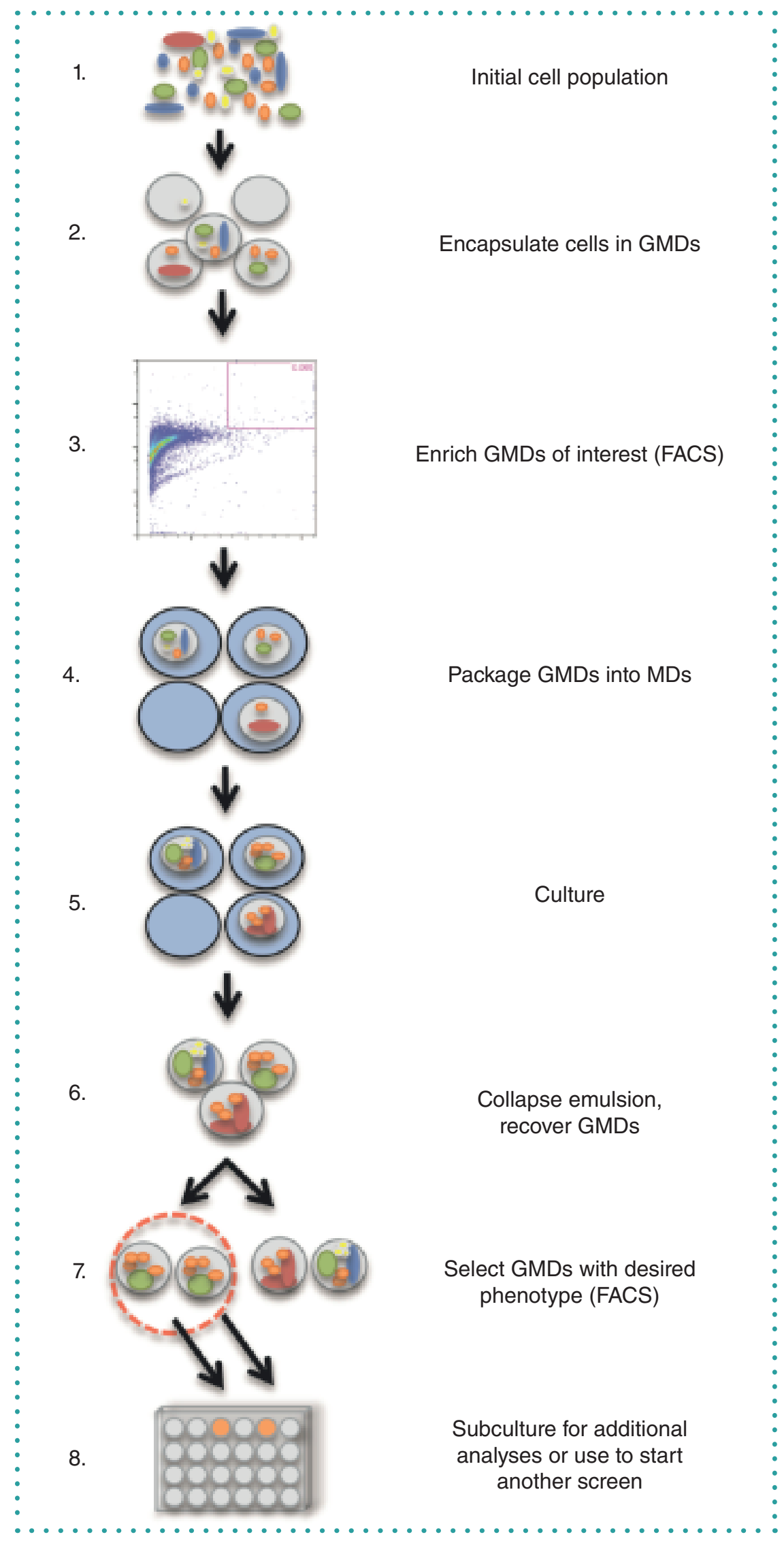

Figure 1. High-throughput screening of cell-to-cell interactions (HiSCl) pipeline workflow. FACS: Fluorescence-activated cell sorting; GMD: Gel microdroplet; MD: Microdroplet. 


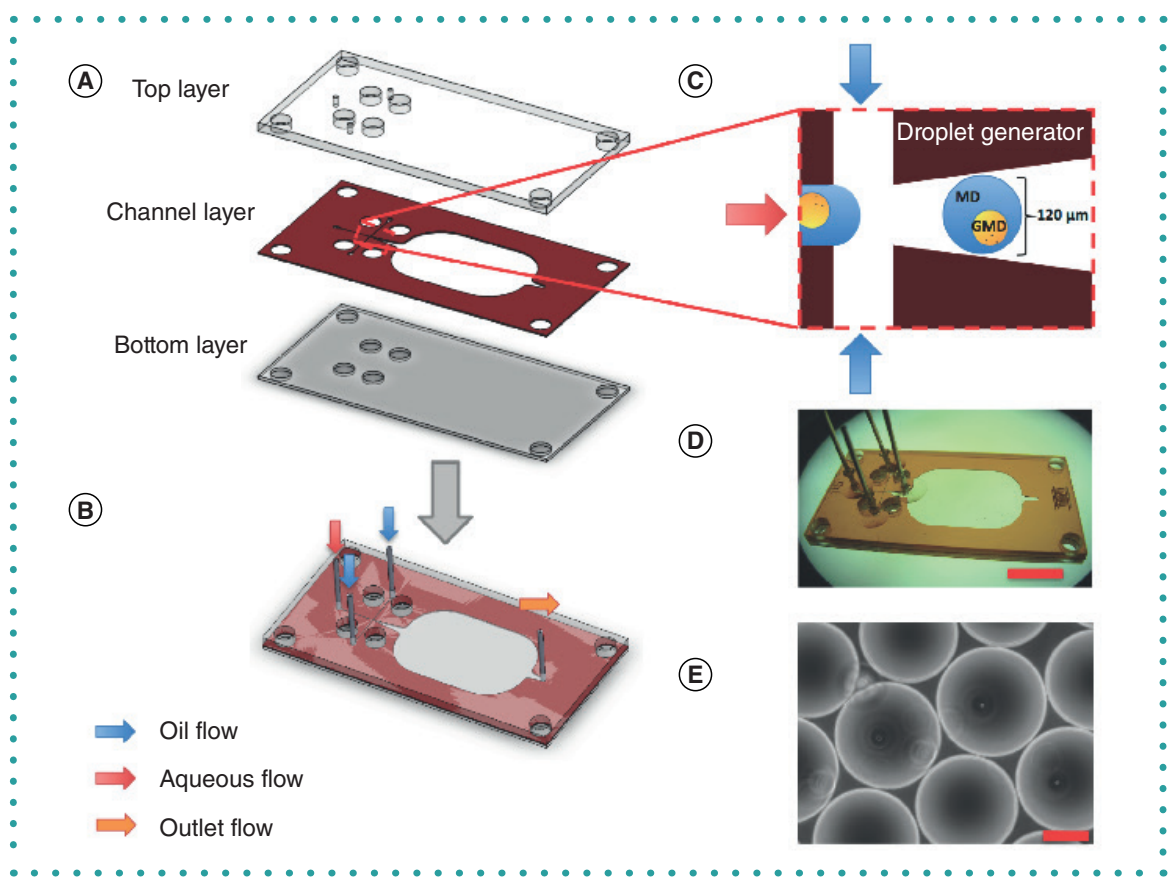

Figure 2. Microfluidic design schematics. The droplet generator was constructed from three layers (A) using a rapid prototyping method that utilizes laser-patterned polymeric films/sheets and lamination. The top and the bottom layers were made of 1.58- $\mathrm{mm}$ thick acrylic sheets, which were laminated with a silicone adhesive transfer tape on one side. The middle layer (channel layer) was made of a $100 \mu \mathrm{m}$-thick polyimide film. The top and the bottom layers were patterned using a $\mathrm{CO}_{2}$ laser cutter. The droplet generation channel geometry (C) was patterned in the middle layer using a UV laser cutter. All three layers were aligned and laminated using a hand roller to complete the fabrication process (B). The top layer had inlet and outlet holes (needles were glued with epoxy to each) to access the droplet (D) generation channels. All devices were treated with a $10 \%$ bleach solution for $15 \mathrm{~min}$ at room temperature and thoroughly flushed with deionized, MilliQ filtered water prior to use. The monodisperse water-in-oil droplets generated (E) were $120 \mu \mathrm{m}$ in diameter. Scale bars are (D) $1 \mathrm{~cm}$ and (E) $50 \mu \mathrm{m}$.

over a period of 60 days (Figure 4). The MDs remained stable and retained their initial size throughout the incubation period. Furthermore, fluorescence was not observed in the dye-free MDs over the course of the incubation, indicating that diffusion of chemicals ( $>700$ Daltons) between MDs is minimal. From these results, we conclude that chemical cross-talk is minimized between bacteria entrapped in separate MDs although we cannot rule out that smaller molecules/metabolites may move out of the aqueous MD into the oil emulsion. Given the long-term stability (> 60 days) of the MDs, the $\mathrm{HiSCl}$ method could, in theory, support the cultivation of slow-growing members of a microbial community. Furthermore, due to the low starting density of cells ( $1-2$ cells per $200 \mu \mathrm{m}$ diameter microdroplet $\sim 2.4 \times 10^{5}$ cells $/ \mathrm{ml})$, compared with a standard flaskbased growth study $\left(5 \times 10^{6} \mathrm{cell} / \mathrm{ml}\right)$ approximately 20 -times more ammonia- $\mathrm{N}$ is available per cell to support growth in HS medium. Based on our calculations, the excess $\mathrm{N}$ availability could feasibly support eight to nine cell doublings compared with four to five doublings observed in a flask experiment.

Finally, to demonstrate direct interrogation of species interactions, algae and bacteria were co-captured and grown in picocultures for several HiSCl iterations $(n=4)$. Chlorophyll autofluorescence was used to measure changes in the size of the algal population in the presence and absence of bacteria after 8-10 days of culturing. After each screening, GMDs containing the largest volume of algae were sorted into culture plates, and were subsampled to determine bacterial community complexity. Sequence analysis (16S rRNA, metagenome) indicated that the bacterial population decreased from 4120 OTUs to only 11 bacteria, from the first to the fourth round of iterative screening, demonstrating reduction of community complexity and enrichment of individual species types (Supplementary Figure 3). The most abundant bacterial species were most similar to Pseudomonas mendocina, Variovorax paradoxus and Ralstonia pickettii (Table 1). Two of these bacteria were successfully isolated from the flow-sorted GMDs by plating on selective growth medium. One isolate, Pseudomonas spp., was then re-introduced to an axenic culture of $C$. sorokiniana 1412 to determine the impact of the bacteria on the growth of the algae. Compared with a pure culture of $C$. sorokiniana $1412, C$. sorokiniana had a faster growth rate $(>30 \%$ improvement) in the presence of Pseudomonas spp. (Figure 5). Recently, bacteria within the Genus Pseudomonas have been shown to be associated with Chlorella [30] and have the ability to modulate the physiology of these Chlorophytes [31-33]. Thus, these data placed in context with the aforementioned studies confirm the efficacy of the HiSCl screening process to identify interspecies interactions.

In this study, we have advanced GMDs' utility beyond the single-cell approach [18-20,34], to a platform that supports high-throughput generation and enrichment of individual GMDs containing multiple microbial species, to interrogate cell-to-cell interactions among defined mixtures of microorganisms. Furthermore, we demonstrated that FACS could be used to overcome a fundamental limitation inherent to many GMD cell-capture protocols - formation of empty GMDs. In addition to separating GMDs with and without microbial cells (Figure 1, step 3), FACS was also used to screen GMDs postcultivation to identify those that supported growth of algal microcolonies (Figure 1, step 7). Sorted and intact GMDs can then be interrogated using microscopy, undergo further cultivation/ growth assays, or be analyzed with various molecular assays (e.g., whole-genome amplification, 16S rRNA phylotyping).

We used a chemically defined minimal growth medium (HS) as the sheath fluid during the enrichment step (Figure 1, step 3). Conceivably, researchers can similarly use a medium as dual purpose for sheath and growth for their specific applications, which then can directly feed into the MD generation process post-enrichment. Additionally, because flow cytometry quantifies the exact number of target GMDs in the growth medium/sheath fluid, it greatly 
enhances the ability to calculate and design packaging conditions required to capture single GMDs in oil MDs for subsequent cultivation. Thus, using growth medium as sheath fluid permits the direct formation of GMD-MDs. However, researchers need to first identify the salt concentration in the intended sheath/growth medium, as salt concentration is a critical parameter for both flow cytometry and algal/bacterial growth.

The time required to generate GMD-MDs depends on both the GMD concentration and the size of MDs that are produced. Varying input flow rates of the aqueous and oil solutions changes the size of MDs. Flow rates of 100/100/50 $\mu \mathrm{l} / \mathrm{min}$ (oil/oil/sample) produced uniform 120- $\mu \mathrm{m}$ (diameter) MDs. An encapsulation period of 43 minutes produced nearly $1.2 \times 10^{7} \mathrm{MDs}$. Under these conditions the percentage of MDs expected to contain a single GMD was calculated as $2.8 \%$, whereas the estimated experimental capture of single GMDs into MDs was $1.7 \%$ (data not shown). Thus, decreasing the rate of capture was necessary to generate MDs containing only one GMD.

We demonstrate that a single algal cell and multiple bacterial cells can be effectively encapsulated in a single GMD, resulting in the growth and viable recovery of microcolonies of algae and bacteria. Hundreds of thousands of candidate GMDs can be cultivated under controlled conditions and screened for robust, moderate or no microcolony growth. Although a number of cell
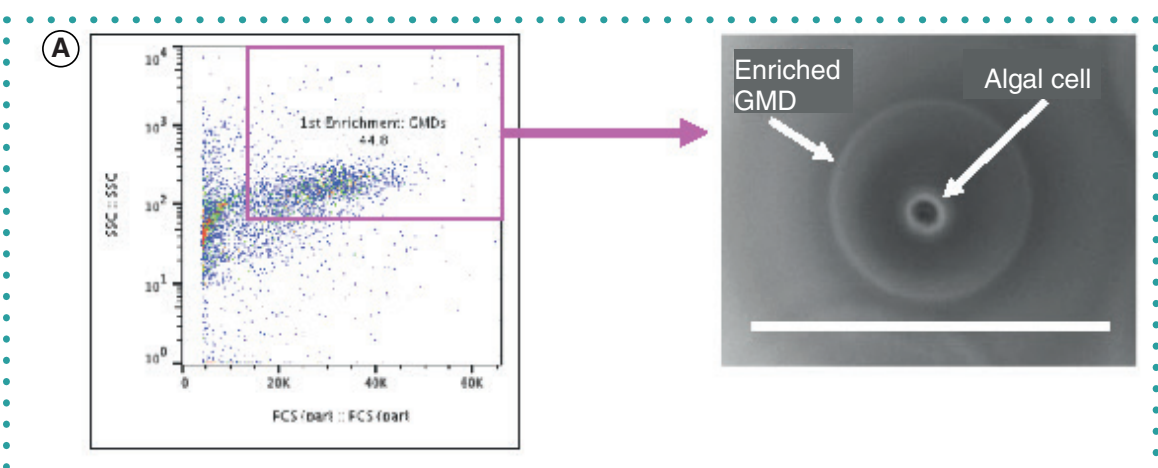

(B)
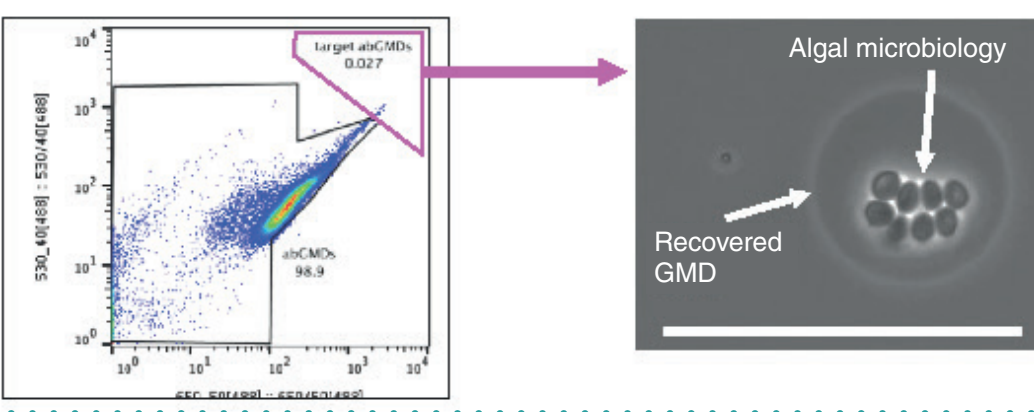

Figure 3. Fluorescence-activated cell sorting enrichment and recovery. (A) Algal/bacterial GMDs (abGMDs) seeded with 1 algal cell and $\sim-5$ environmental bacteria (i.e., Figure 1, step 3 ) and (B) abGMDs containing microcolonies arising from replication of algae during the cultivation stage in oil (i.e. Figure 1, step 7). Scale bar: $50 \mu \mathrm{m}$.

GMD: Gel microdroplet.

separation technologies exist (e.g., micromanipulator, laser tweezer), none provide the high-throughput analytical capabilities of flow cytometry. In this study, we recovered $\sim 110,000$ GMDs per iteration, which were screened via flow cytometry in minutes. Our selection strategy utilized the combination of a priori knowledge of GMDs that likely contained algal/bacterial cells of relative densities (i.e., SSC) and chlorophyll autofluorescence. Our gating strategy greatly improved the ability to identify the recovered GMDs that contained algal microcolonies (e.g., Figure 3B) and discern the GMDs that did not possess this phenotype (e.g., Figure 3B, abGMDs outside of the gate). This is specifically evidenced by the fact that no candidate GMDs possessing microcol-

\section{Table 1. Relative abundance of identified bacteria using read-based analysis after four rounds of high-throughput screening of cell-to-cell interactions.}

\begin{tabular}{|l|l|l|}
\hline Species & Abundance (\%) & Accession \\
\hline Ralstonia pickettii & 46.3 & MK291417 \\
\hline Variovorax paradoxus & 10 & MK291415 \\
\hline Pseudomonas spp. & 8.4 & MK291416 \\
\hline Propionibacterium acnes & 7.7 & MK434321 \\
\hline Comamonas testosteroni & 7.1 & MK434324 \\
\hline Sphingomonas sp. & 5.4 & MK434315-6 \\
\hline Bradyrhizobium sp. & 3.4 & MK434319 \\
\hline Methylobacterium spp. & 3.4 & MK434322 \\
\hline Stenotrophomonas maltophilia & 3.2 & MK434320 \\
\hline Pseudoalteromonas sp. & 3.2 & MK434317-8 \\
\hline Sphingopyxis alaskensis & 1.9 & MK434323 \\
\hline
\end{tabular}




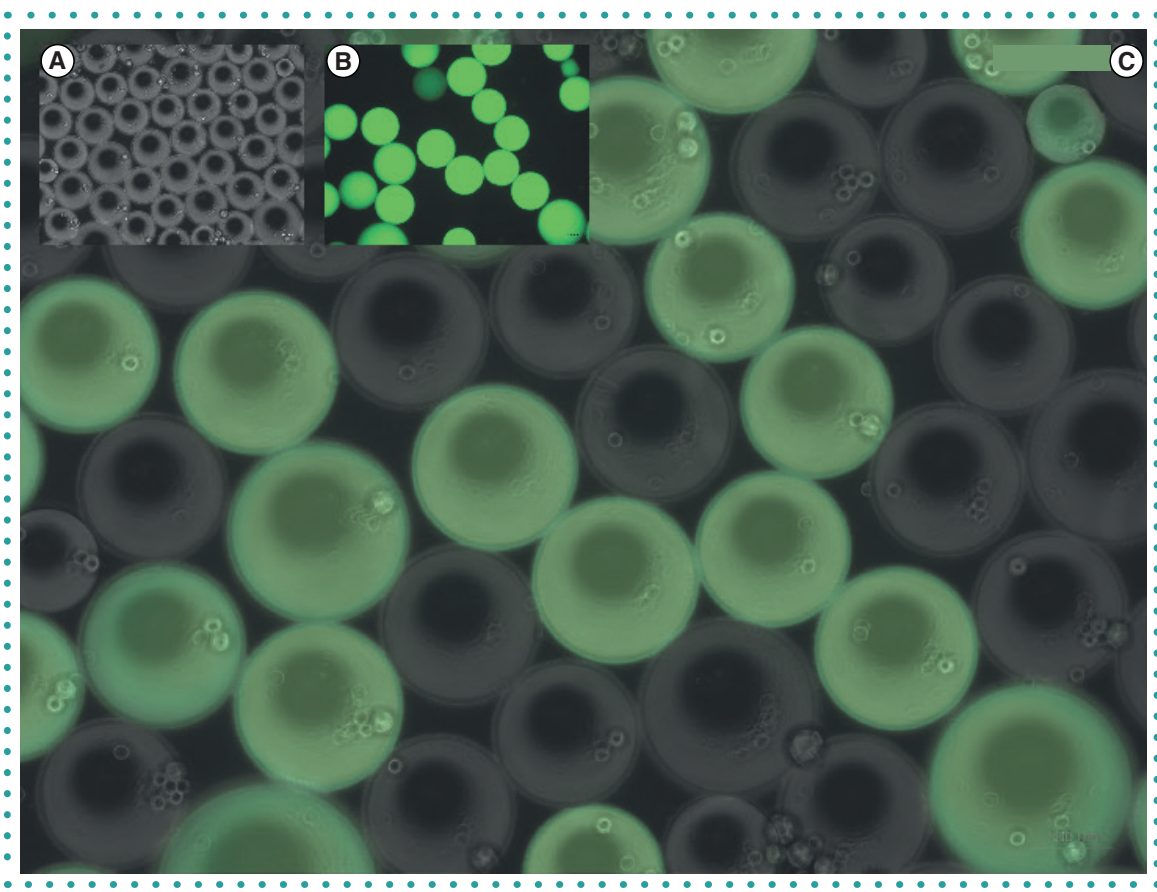

Figure 4. Stability of microdroplets (oil phase, HFE-7500, Krytox) injected with either high salt growth medium alone or high salt medium containing the fluorescent dye, Alexa Fluor 532 , following a 60-day incubation period in a cultivation chamber at room temperature. Images from (A) brightfield microscopy, (B) fluorescence microscopy, and (C) a brightfield/fluorescence overlay. Scale bar (top right): $100 \mu \mathrm{m}$.

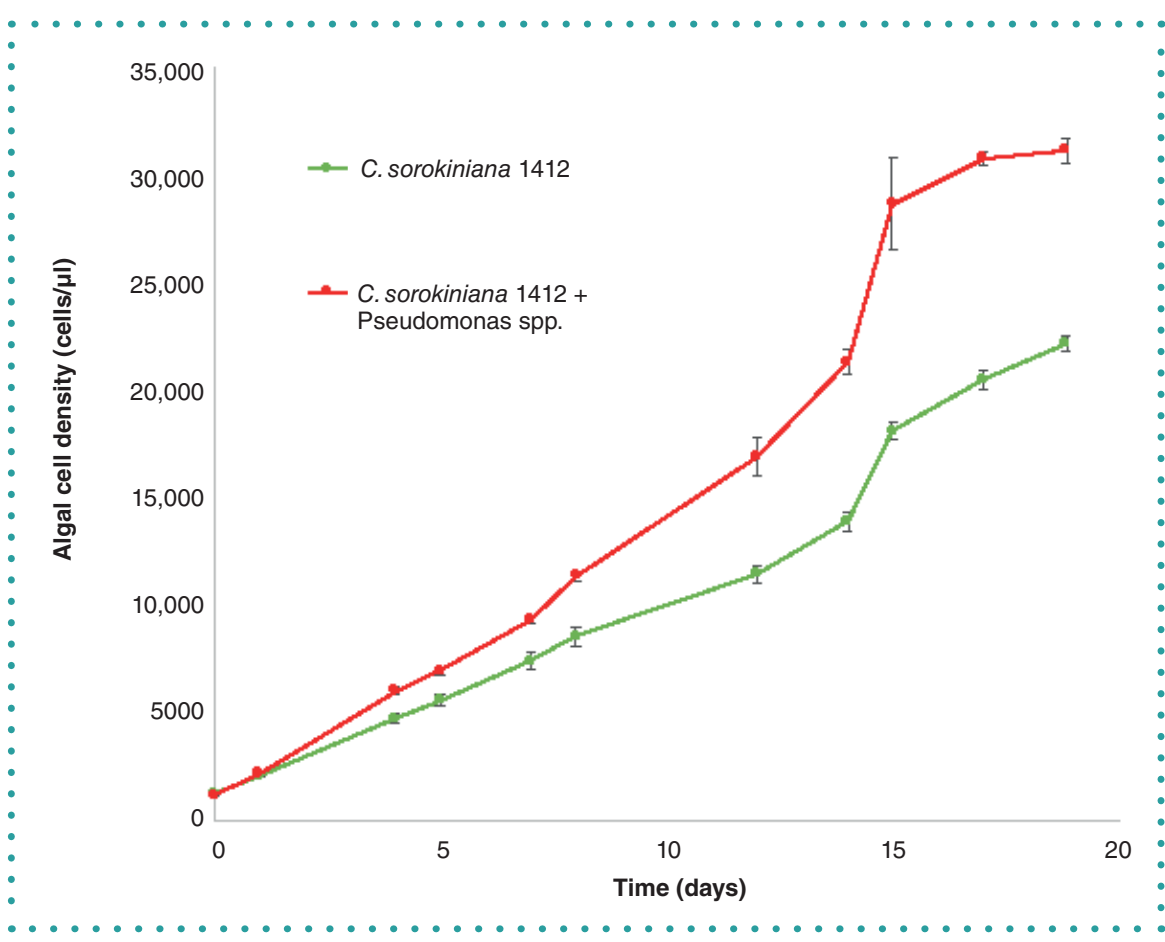

Figure 5. Growth enhancement of Chlorella sorokiniana from isolate bacteria. C. sorokiniana was grown with and without Pseudomonas spp. Pseudomonas was seeded at a 25:1 ratio of bacterial cells to algal cells. Error bars show standard error of five replicates.
$>$ onies were present in the cultivated control aGMD population.

In summary, we have demonstrated that multiple rounds of HiSCl selection can successfully isolate members of a complex bacterial input population that induce a desired phenotype (i.e., augmentation of growth rates), thus verifying our hypothesis. Furthermore, by recovering intact, viable cells with flow cytometry, the Hiscl pipeline supports several downstream applications, such as advance phenotypic analysis, sequencing, or potentially long-term cultivation, which cannot be performed using picoliter volumes of cell suspensions.

\section{FUTURE PERSPECTIVE}

Although significant progress was made toward interrogating complex microbial communities with a microfluidic-based cultivation and screening platform, additional improvements to the HiSCl platform will enable more efficient screening and characterization of cell-cell interactions. Ideally, the HiSCl platform will evolve to enable all steps of the workflow to be conducted 'on chip'. First, GMDs can conceivably be created on chip and inertial focusing could be exploited to enable ordered packaging of cells within GMDs and loading of GMDs into MDs. Second, on-chip cell sorting and phenotyping could also be integrated to further improve screening efficiency and would virtually eliminate the need for encapsulation in GMDs. Furthermore, we envision that a variety of detectors could be built into the platform to enable the use of common fluorescence or colorimetric assays that measure many types of cellular responses beyond changes in growth rates (i.e., changes in lipid bodies, viability, enzymatic activity, toxin production, pathogen sensitivity, nutrient uptake rates, etc). In summary, a complete on-chip $\mathrm{HiSCl}$ platform will decrease preparation time, greatly increase the number of cell-to-cell interactions that can be screened, and expand the number of phenotypes that could be measured simultaneously.

\section{AUTHOR CONTRIBUTIONS}

SRS, MV, PN and AED conceived, developed and executed the study and technique. JO, $\mathrm{BP}$, and $\mathrm{BH}$ and $\mathrm{AED}$ contributed to the experimental design and analysis and provided technical assistance. JHH contributed 
technical assistance towards the design and fabrication of the microfluidic devices. All authors contributed to the writing and editing of the manuscript.

\section{FINANCIAL \& COMPETING INTERESTS DISCLOSURE}

This work was funded by Los Alamos National Laboratory through a Laboratory Directed Research and Development Award \# 20140216ER and award DE-EE0008122 from the Bioenergy Technologies Office with the Department of Energy. The authors have no other relevant affiliations or financial involvement with any organization or entity with a financial interest in or financial conflict with the subject matter or materials discussed in the manuscript apart from those disclosed.

No writing assistance was utilized in the production of this manuscript.

\section{OPEN ACCESS}

This work is licensed under the AttributionNonCommercial-NoDerivatives 4.0 Unported License. To view a copy of this license, visit http://creativecommons.org/licenses/ by-nc-nd/4.0/

\section{SUPPLEMENTARY DATA}

To view the supplementary data that accompany this paper please visit the journal website at: www.future-science. com/doi/suppl/10.2144/btn-2018-0124

\section{REFERENCES}

1. Council NR. The new science of metagenomics: revealing the secrets of our microbial planet. National Academies Press (2007)

2. Lewis $K$, Epstein S, D'onofrio A, Ling LL. Uncultured microorganisms as a source of secondary metabolites. J. Antibiot. 63(8), 468 (2010).
3. Kojima T, Nagao N, Ando D et al. Emulsion culture: a miniaturized library screening system based on micro-droplets in an emulsified medium. J. Biosci. Bioeng. 112(3), 299-303 (2011).

4. Lagus TP, Edd JF. High-throughput co-encapsulation of self-ordered cell trains: cell pair interactions in $\mathrm{mi}$ crodroplets. Rsc Adv. 3(43), 20512-20522 (2013).

5. Schoeman RM, Kemna EW, Wolbers F, Van Den Berg A. High-throughput deterministic single-cell encapsulation and droplet pairing, fusion, and shrinkage in a single microfluidic device. Electrophoresis 35(2-3), 385-392 (2014).

6. Kemna EW, Schoeman RM, Wolbers F, Vermes I, Weitz DA, Van Den Berg A. High-yield cell ordering and deter ministic cell-in-droplet encapsulation using Dean flow in a curved microchannel. Lab Chip 12(16), 2881-2887 (2012).

7. Abate AR, Hung T, Mary P, Agresti JJ, Weitz DA High-throughput injection with microfluidics using picoinjectors. Proc. Natl Acad. Sci. USA 107(45) 19163-19166 (2010).

8. Mazutis L, Gilbert J, Ung WL, Weitz DA, Griffiths AD, Heyman JA. Single-cell analysis and sorting using droplet-based microfluidics. Nat. Protoc. 8(5), 870 (2013)

9. Kim HS, Guzman AR, Thapa HR, Devarenne TP, Han A. A droplet microfluidics platform for rapid microalgal growth and oil production analysis. Biotechnol. Bioeng 113(8), 1691-1701 (2016).

10. Kim HS, Hsu SC, Han SI et al. High-throughput droplet microfluidics screening platform for selecting fast-growing and high lipid-producing microalgae from mutant library. Plant Direct 1(3), e00011 (2017).

11. Duffy DC, Mcdonald JC, Schueller OJ, Whitesides GM. Rapid prototyping of microfluidic systems in poly (di-

. Nath P, Fung D, Kunde YA, Zeytun A, Branch B, Goddard $G$. Rapid prototyping of robust and versatile microfluidic components using adhesive transfer tapes. Lab Chip 10(17), 2286-2291 (2010)

13. Zinchenko A, Devenish SR, Kintses B, Colin P-Y, Fischlechner $M$, Hollfelder $F$. One in a million: flow cytometric sorting of single cell-lysate assays in monodisperse picolitre double emulsion droplets for directed evolution. Anal. Chem. 86(5), 2526-2533 (2014).

14. One Cell Systems, Inc., Gel Microdrop Assay Systems, GMD Bacterial Growth Assay: Experimental Guidelines and Protocols. MA, USA (2003)

15. Zengler $\mathrm{K}$, Toledo $\mathrm{G}$, Rappé $\mathrm{M}$ et al. Cultivating the uncultured. Proc. Natl Acad. Sci. USA 99(24), 15681-15686 (2002).

16. Chen W, Kim J-H, Zhang D et al. Microfluidic one-step synthesis of alginate microspheres immobilized with antibodies. J. Royal Soc. Interface 10(88), 20130566 (2013).

17. Lian M, Collier CP, Doktycz MJ, Retterer ST. Monodisperse alginate microgel formation in a three-dimensional microfluidic droplet generator. Biomicrofluidics 6(4) 044108 (2012).

18. Dichosa AE, Davenport KW, Li P-E et al. Draft genome sequence of Thauera sp. strain SWB20, isolated from a Singapore wastewater treatment facility using gel microdroplets. Genome Announc. 3(2), e00132-00115 (2015).

19. Fitzsimons MS, Novotny M, Lo C-C et al. Nearly finished genomes produced using gel microdroplet culturing reveal substantial intraspecies genomic diversity within the human microbiome. Genome Res. 23(5), 878-888 (2013).
20. Dichosa AE, Daughton AR, Reitenga KG, Fitzsimons MS, Han CS. Capturing and cultivating single bacterial cells in gel microdroplets to obtain near-complete genomes. Nat. Protoc. 9(3), 608 (2014).

21. Hosokawa M, Hoshino Y, Nishikawa Y et al. Droplet-based microfluidics for high-throughput screening of a metagenomic library for isolation of microbial

2. Eun Y-J, Utada AS, Copeland MF, Takeuchi S, Weibel DB. Encapsulating bacteria in agarose microparticles using microfluidics for high-throughput cell analysis and isolation. ACS Chem. Biol. 6(3), 260-266 (2010).

23. Griffiths MJ, Harrison ST. Lipid productivity as a key characteristic for choosing algal species for biodiese production. J. Appl. Psychol. 21(5), 493-507 (2009).

24. Caporaso JG, Lauber CL, Walters WA et al. UItra-high-throughput microbial community analysis on
the Illumina HiSeq and MiSeq platforms. ISME J. 6(8), 1621 (2012).

25. Kozich JJ, Westcott SL, Baxter NT, Highlander SK Schloss PD. Development of a dual-index sequencing strategy and curation pipeline for analyzing amplicon sequence data on the MiSeq Illumina sequencing platform. Appl. Environ. Microbiol. 79(17), 5112-5120 (2013).

26. Sueoka N. Mitotic replication of deoxyribonucleic acid in Chlamydomonas reinhardi. Proc. Natl Acad. Sci. USA 46(1), 83-91 (1960).

27. Caporaso JG, Kuczynski J, Stombaugh J et al. QIIME allows analysis of high-throughput community sequencing data. Nat. Methods 7(5), 335-336 (2010).

28. Desantis $T Z$, Hugenholtz $P$, Larsen $N$ et al. Greengenes, a chimera-checked 16S rRNA gene database and workbench compatible with ARB. Appl. Environ. Microbiol. 72(7), 5069-5072 (2006).

29. Freitas TAK, Li P-E, Scholz MB, Chain PS. Accurate read-based metagenome characterization using a hierarchical suite of unique signatures. Nucleic Acids Res. 43(10), e69 (2015).

30. Bell TA, Prithiviraj B, Wahlen BD, Fields MW, Peyton BM. A lipid-accumulating alga maintains growth in outdoor, alkaliphilic raceway pond with mixed mict
nities. Front. Microbiol. 6, 1480 (2016).

31. De-Bashan LE, Hernandez J-P, Morey T, Bashan Y Microalgae growth-promoting bacteria as 'helpers' for microalgae: a novel approach for removing ammonium and phosphorus from municipal wastewater. Water Res. 38(2), 466-474 (2004).

32. Afi L, Metzger P, Largeau C, Connan J, Berkaloff C Rousseau $B$. Bacterial degradation of green microalgae: incubation of Chlorella emersonii and Chlorella vulgaris with Pseudomonas oleovorans and Flavobacterium aquatile. Org. Geochem. 25(1-2), 117-130 (1996).

33. Cheah WY, Show PL, Juan JC, Chang J-S, Ling TC. Waste to energy: the effects of Pseudomonas sp. on Chlorella sorokiniana biomass and lipid productions in palm oil mill effluent. Clean Technol. Envir. 1-9 (2018).

34. Dichosa AE, Fitzsimons MS, Lo C-C et al. Artificial polyploidy improves bacterial single cell genome recovery. PLoS One 7(5), e37387 (2012). 